\title{
Reachability Analysis of a Biodiesel Production System Using Stochastic Hybrid Systems
}

\author{
Derek Riley, Xenofon Koutsoukos \\ ISIS/EECS Vanderbilt University \\ Nashville, TN 37209, USA \\ Derek.Riley, Xenofon.Koutsoukos@vanderbilt.edu
}

\author{
Kasandra Riley \\ Mayo Foundation \\ Rochester, MN 55905, USA \\ riley.kasandra@mayo.edu
}

\begin{abstract}
Modeling and analysis of chemical reactions are critical problems because they can provide new insights into the complex interactions between systems of reactions and chemicals. One such set of chemical reactions defines the creation of biodiesel from soybean oil and methanol. Modeling and analyzing the biodiesel creation process is a challenging problem due to the highly-coupled chemical reactions that are involved. In this paper we model a biodiesel production system as a stochastic hybrid system, and we present a probabilistic verification method for reachability analysis. Our analysis can potentially provide useful insights into the complicated dynamics of the chemicals and assist in focusing experiments and tuning the production system for efficiency. The verification method employs dynamic programming based on a discretization of the state space and therefore suffers from the curse of dimensionality. To verify the biodiesel system model we have developed a parallel dynamic programming implementation that can handle large systems. Although scalability is a limiting factor, this work demonstrates that the technique is feasible for realistic biochemical systems.
\end{abstract}

\section{INTRODUCTION}

Modeling and analysis of chemical reactions are important tasks because they can unlock insights into the complicated dynamics of systems which are difficult or expensive to test experimentally. A variety of techniques have been used to model chemical equations, but the effectiveness of the analysis techniques is often limited by tradeoffs imposed by the modeling paradigms. Stochastic differential equations have been used to model biochemical reactions [11], [3]; however, analysis of these models has mainly been limited to simulation. Hybrid systems have also been used [2], [10]; however, hybrid systems do not capture the probabilistic nature inherent in chemical reactions, and therefore, may not be able to correctly analyze certain systems. Stochastic Hybrid Systems (SHS) have been used to capture the stochastic nature of chemical systems but have previously only been used for simulations [22] or analysis of systems with simplified continuous dynamics [13].

In this paper we analyze the biochemical process of creating biodiesel. Biodiesel is created by transesterification of large, branched triglycerides from soybean oil into smaller, strait-chain molecules of methyl esters (biodiesel) using methanol and lye in a well-mixed, heated processor [23]. If incorrect proportions of methanol are used, the reaction will not complete and the biodiesel will not pass purity tests. Also, if too much methanol is used, the methanol must be recovered later which adds time and cost to the process.
There are two main types of systems which are intended to produce biodiesel: batch and continuous. Continuous processors are generally large commercial machines which process the ingredients and produce biodiesel in a continuous flow. Batch systems are more common because they are simpler and cheaper to construct. The idea of a batch system is to combine all the necessary chemicals in a single vessel with a heater and mixing system and produce one batch at a time. In this work we model the batch style processor presented in [8].

Our model of the biodiesel reactions also incorporates the continuous temperature of the reacting solution and a thermostat-controlled heater. Temperature is a major factor in the rate at which chemicals react because it affects the kinetic energy of the individual molecules. As temperature of a mixture of chemicals increases, the chemicals react more often because of the increased kinetic energy. Likewise, as temperature decreases, the reduction in kinetic energy decays the reaction rates in a predictable manner.

The chemical master equation accurately models the stochastic dynamics for chemical reactions, but it is impossible to solve for most practical systems [11]. The Stochastic Simulation Algorithm (SSA) is equivalent to solving the master equation based on a discrete model by simulating one reaction at a time, but if the number of molecules of any of the reactants is large, the SSA is not efficient [22]. It is computationally intractable to enumerate all possible states of the model employed by the SSA for formal verification because the reaction rates depend on the concentrations and the SSA models individual molecules. Therefore, our approach suggests starting with the continuous stochastic dynamics and generating discrete approximations with coarser (and variable) resolution unlike the fixed, overly-fine resolution of the SSA. The discrete approximations can then be used for verification of reachability properties [17], [18].

We model the biodiesel production equations using SHS and use a dynamic programming verification method based on a discretization of the state space. The contribution of the paper centers on the application of the theoretical results presented in [17], [18] to a biodiesel production system, a realistic and important biochemical process. Our results demonstrate that SHS are wellsuited for modeling and verification of such biochemical processes. The proposed method suffers from the curse of dimensionality. Therefore, we have developed a parallel dynamic programming implementation of the verifica- 
tion algorithm that can handle large systems. This work demonstrates that the technique is feasible for realistic systems of chemical equations even though scalability is a limiting factor.

The organization for the rest of the paper is as follows: Section 2 describes the related work, Section 3 describes SHS modeling of systems of chemical equations for biodiesel, Section 4 describes the probabilistic verification method, Section 5 presents our experimental results, and Section 6 concludes the work.

\section{RELATED WORK}

Biodiesel reactions have been previously modeled using differential equations under constant temperature conditions [8], [20]. A kinetic-based modeling technique for the biodiesel reactions is presented in [1]. Different biodiesel processor designs and processing techniques are compared in [24]. Since biodiesel processors involve switches, pumps, and variable temperatures, hybrid modeling techniques can be used to accurately model the real processors.

Hybrid systems have been used for modeling biological-based systems in order to capture the complicated dynamics using well-defined abstractions. Biological protein regulatory networks have been modeled with hybrid systems using linear differential equations to describe the changes in protein concentrations and discrete switches to activate or deactivate the continuous dynamics based on protein thresholds [10]. Stochastic hybrid systems further improve on the benefits of hybrid systems by providing a probabilistic framework for realistically modeling chemical reactions. A modeling technique that uses SHS to construct models for chemical reactions involving a single reactant specie is presented in [13]. A genetic regulatory network was modeled with a SHS model and compared to a deterministic model in [15]. SHS models of biochemical systems have been developed and simulated using hybrid simulation algorithms in [12], [22].

This paper adopts a SHS model that is a special case of the general model presented in [6] and employs a reachability analysis method based on discrete approximations. Discrete approximation methods based on finite differences have been studied extensively in [19]. Based on discrete approximations, the reachability problem can be solved using algorithms for discrete processes [21]. The approach has been applied for optimal control of SHS given a discounted cost criterion in [16]. For verification, the discount term cannot be used and convergence of the value function can be ensured only for appropriate initial conditions. A related grid based method for safety analysis of stochastic systems with applications to air traffic management has been presented in [14]. Our approach is similar but using viscosity solutions we show the convergence of the discrete approximation methods.

Reachability analysis for SHS can also be performed using Monte Carlo methods [5]. Multiple stochastic simulations are used to determine the reachability probability for an initial state of a SHS. Confidence intervals and accuracy probabilities can be selected by adjusting the number of simulations.

\section{Modeling Chemical Reactions Using SHS}

\section{A. Dynamics of Chemical Reactions}

A chemical reaction specifies all chemical species which react (reactants) and are produced (products). A kinetic constant $k$, associated with each reaction, numerically describes the affinity for the reactants to produce the products in constant temperature conditions. Experimental analysis is used to physically measure the variation in individual concentrations of the chemical species in a biochemical system. However, understanding the dynamical behavior of biochemical systems requires running many experiments that can be time consuming, tedious, unsafe, or costly. Developing and analyzing dynamical models for capturing the evolution of individual chemical species concentrations can reduce the number of experiments needed.

Chemical reactions are inherently probabilistic because of the unpredictability of molecular motion [9], so their dynamics can be accurately described by stochastic models. Discrete stochastic models of reactions can be created by describing a reaction $j$ as firing at a rate $a_{j}$ [7]. When the reaction fires, the concentrations of the reactants and products are reset to the appropriate updated values. Table I shows the rates and resets for several examples of different types of reactions. For example, when the reaction $X \rightarrow Z$ occurs, a molecule of $X$ is consumed and a molecule of $Z$ is produced denoted by $x-=1$ and $z+=1$ respectively where $x$ and $z$ are the quantities of molecules of chemical species $X$ and $Z$, and $k_{i}$ is the kinetic constant for reaction $i$.

\begin{tabular}{|c|c|c|}
\hline Reaction & $a_{j}$ & Reset \\
\hline$X \rightarrow Z$ & $k_{1} x$ & $x-=1 ;$ \\
$X+Y \rightarrow 2 Z$ & $k_{2} x y$ & $x-=1 ;$ \\
& & $y-=1 ;$ \\
& & $z+=2 ;$ \\
$2 X \rightarrow Z$ & $1 / 2 * k_{3} x(x-1)$ & $z+=1 ;$ \\
$2 X+Y \rightarrow 2 Z$ & $1 / 2 * k_{4} x(x-1) y$ & $x-=2 ;$ \\
& & $y-1 ;$ \\
$3 X \rightarrow Z$ & $1 / 6 * k_{5} x(x-1)(x-2)$ & $x-=3 ;$ \\
& \multicolumn{2}{|c}{} \\
& & $z+=1 ;$ \\
\hline
\end{tabular}

TABLE I

EXAMPLE REACTION RATES AND RESETS

Discrete modeling is ideal for small systems with low concentrations, but systems with a large number of molecules quickly become inefficient to analyze. The dynamics of these large systems can, however, be described using stochastic differential equations assuming that the reactions happen in a well mixed solution.

Continuous modeling of chemical reactions can be accomplished using the following technique. Suppose that we have a system of $M$ chemical reactions and $N$ chemical species. We define $x_{i}$ as the concentration of 
the $i$ th chemical species in micro-Molarity $(\mu \mathrm{M}), M_{f}$ as the number of reactions, $a_{j}$ as the reaction propensity of the $j$ th reaction, and $W$ as an $M_{f}$-dimensional Wiener process. Reaction propensities are calculated using the kinetic constants and concentrations of the reactants for each chemical reaction. The stoichiometric matrix $v$ is a $\left(M_{f} \mathrm{X} N\right)$ matrix whose values represent the concentration of chemical species lost or gained in each reaction $(0,+1,-1,+2$, etc. $)$. Equation (1) describes the continuous dynamics for each of the $i$ chemical species [22].

$$
d x_{i}=\sum_{j=1}^{M_{f}} v_{j i} a_{j}(x(t)) d t+\sum_{j=1}^{M_{f}} v_{j i} \sqrt{a_{j}(x(t))} d W_{j}
$$

All chemical reaction rates are effected by the temperature at which they occur. The higher the temperature, the more likely that the individual molecules will interact and eventually react. The chemical reaction rate $k$ is most often defined for a single temperature and pressure, but most chemical reactions are exothermic or endothermic and therefore inherently change the temperature.

Furthermore, it is advantageous to control the reaction rates by applying or removing heat to ensure that the system behaves correctly. The effect of temperature on the reaction rate, $k$, is given by $k=A e^{\frac{-E a}{R T}}$ where $A$ is a constant for each reaction, $E a$ is the activation energy for each reaction, $R$ is the gas constant (1.9872), and $T$ is the temperature in Kelvin (for example see [8]). Using this equation we can determine the reaction rates for each reaction at any temperature and therefore model the fluctuating reaction speeds.

A heating or cooling apparatus generally applies heat or cool in a binary manner (on or off), so a discrete model of heating control is necessary. Stochastic hybrid systems are ideal for modeling systems of chemical reactions because they are able to model continuous and discrete dynamics in a stochastic framework. Temperature can easily be included in a stochastic model as another continuous state. The temperature can then be used to help calculate the reaction rates for the individual reactions.

\section{B. Biodiesel Production}

Biodiesel can be produced by combining soybean oil, methanol, and lye under the correct conditions [20]. The lye is used to neutralize free fatty acids, and is a fairly simple component of the biodiesel process, so we assume that the system has no free fatty acids so we can focus on the more complicated reactions.

Once the free fatty acids have been neutralized, the soybean oil is comprised mainly of triglycerides (TG). The TG can be mixed with methanol (M) and turned into biodiesel in a process called transesterification. The chemicals involved in the transesterification process are described in Table II and the reactions are described in Table III. The TGs are turned into diglycerides (DGs) which are turned into monoglycerides (MGs) which are turned into esters (E) also known as biodiesel. The only byproduct of the reaction is glycerol $(\mathrm{Gl})$ which can be used as a hand soap. We model the concentration of each of these six chemical species as a continuous variable in our model. Each of the six reactions are modeled using the SDE (1). The kinetic values are determined by the equations in Table III [20].

\begin{tabular}{|c|c|c|c|}
\hline Reactant & Variable & {$[$ Min, Max] (Moles) } & Resolution \\
\hline$T G$ & $x_{1}$ & {$[0.00001,4.00001]$} & 0.2 \\
$D G$ & $x_{2}$ & {$[0.00001,4.00001]$} & 0.4 \\
$M G$ & $x_{3}$ & {$[0.00001,4.00001]$} & 0.4 \\
$E$ & $x_{4}$ & {$[0.00001,4.00001]$} & 0.4 \\
$M$ & $x_{5}$ & {$[0.00001,1.00001]$} & 0.05 \\
$G l$ & $x_{6}$ & {$[0.00001,1.00001]$} & 0.1 \\
$T$ & $x_{7}$ & {$[20,70]$} & 10 \\
\hline
\end{tabular}

TABLE II

CONTINUOUS VARIABLES

\begin{tabular}{|c|c|}
\hline Reaction & Kinetic Rate \\
\hline$T G+M \rightarrow D G+E$ & $k_{1}=3.92 \times 10^{7} e^{\frac{-13145}{1.987 T}}$ \\
$D G+E \rightarrow T G+M$ & $k_{2}=5.58 \times 10^{5} e^{\frac{-9932}{1.987 T}}$ \\
$D G+M \rightarrow M G+E$ & $k_{3}=5.89 \times 10^{13} e^{\frac{-19860}{1.987 T}}$ \\
$M G+E \rightarrow D G+M$ & $k_{4}=9.87 \times 10^{9} e^{\frac{-14369}{1.987 T}}$ \\
$M G+M \rightarrow G L+E$ & $k_{5}=5350 e^{\frac{-6421}{1.987 T}}$ \\
$G L+E \rightarrow M G+M$ & $k_{6}=21500 e^{\frac{-9588}{1.987 T}}$ \\
\hline
\end{tabular}

TABLE III

BIODIESEL REACTIONS AND KINETIC EQUATIONS

The chemical reactions involved in the biodiesel production process are affected greatly by temperature, but previous models of the biodiesel reactions have assumed a constant temperature. Since more heat added to the system will increase the reaction rates $(k)$, the warmer the reacting chemicals can be, the faster biodiesel will be produced. However, the energy required to heat the system is a major cost of producing biodiesel, so it is important to know if a heating control system will produce biodiesel successfully under realistic conditions.

The model we have developed uses temperature $(T)$ as another continuous state of the system. We use the temperature to calculate the kinetic constants $(k)$ for each reaction as seen in Table III. The biodiesel reactions create a negligible amount of heat, so we assume that the only heat added to the system comes from a heating element. We also assume that when the heating element is not on, the system loses heat at a constant rate.

$$
\dot{x_{7}}=\left\{\begin{array}{cc}
.02\left(-x_{7}\right) d t+.01 d W & \text { cooling } \\
.05\left(100-x_{7}\right) d t+.01 d W & \text { heating }
\end{array}\right.
$$

We model the change in heating using two discrete states, one for heating and one for cooling. The continuous dynamics of the two states are determined by combining the equations (1) describing the rate of concentration change with the equations in Table III describing the effect of temperature on the kinetic constants. Furthermore, the temperature is described as another continuous state of the system where the continuous dynamics for $\dot{x}_{7}$ are 
described by Equation 2. The system can switch between the states depending on the temperature of the reactants. In this model, if the temperature is above 30 degrees, the heater is turned off, and if the temperature is below 30 degrees the heater is turned on.

Methanol (M) is an expensive chemical necessary for producing biodiesel from soybean oil, and recovery of methanol from the resulting biodiesel can be costly, so conservation of the chemical is necessary. However, having too little methanol in the mix can leave unconverted TGs, DGs, or MGs which will cause the biodiesel to fail quality testing. Therefore, ideally we would like to use our model to test whether all of the TGs, DGs, MGs, and methanol are used up at the same time to ensure quality and efficiency.

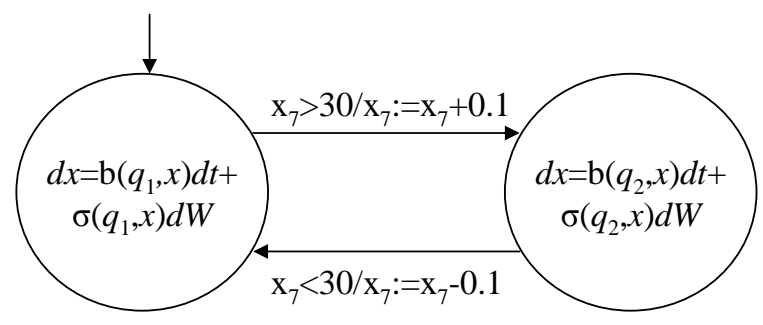

Fig. 1. SHS model of biodiesel production system

The SHS model for biodiesel production is shown in Figure 1. Between transitions, the continuous state evolves according to the corresponding SDE where the solution is understood using the Itô stochastic integral. Upon occurrence of a transition, the continuous state $x$ is reset according to the reset map. A guarded transition fires the instant when the guard becomes true and is reset according to the reset map. We include a reset on the transition to ensure that Zeno behavior is avoided by the model [18].

The functions $b(q, x)$ and $\sigma(q, x)$ shown in Figure 1 come from Equations 1,2 and are bounded and Lipschitz continuous in $x \in X$ and thus the SDE has a unique solution. As described in Table II, the concentrations of the biodiesel production system are assumed to be bounded. Given these assumptions, the SHS for the biodiesel production system is a special case of the SHS model described in [17]. In particular, this model has two discrete states and two guarded discrete transitions.

Our goal of the analysis of the biodiesel model is to determine the probability that the reaction will fully complete with a small excess of methanol. To determine this, we define the set of reachable states as the set of all concentrations that satisfy $T=\left\{x \in \mathbb{R}^{7}: x_{5}<.1 \wedge x_{1}<1 \wedge x_{2}<1 \wedge x_{3}<1\right\}$. Since we don't want the system to run out of TGs, DGs, or MGs before it runs out of methanol, we define the unsafe states as those which satisfy $U=$ $\left\{x \in \mathbb{R}^{7}: x_{5}<.1 \wedge\left(x_{1}>1 \vee x_{2}>1 \vee x_{3}>1\right)\right\}$. Our problem is to determine what is the probability that the SHS will enter the reachable set without entering the unsafe set.

\section{Probabilistic Verification}

\section{A. Reachability Analysis}

Given a target set and an unsafe set of states, the objective of the reachability problem is to compute the probability that the system execution from an arbitrary initial state will reach the target set while avoiding the unsafe set. We denote $X^{q}$ the state space for mode $q$ and $S=\cup_{q} X^{q}$ the state space of the system. Let $T=\cup_{q \in Q_{T}}\{q\} \times T^{q}$ and $U=\cup_{q \in Q_{U}}\{q\} \times U^{q}$ the set of target and unsafe states respectively. We assume that $T^{q}$ and $U^{q}$ are proper open subsets of $X^{q}$ for each $q$, i.e. $\partial T^{q} \cap \partial X^{q}=\partial U^{q} \cap \partial X^{q}=\emptyset$ and the boundaries $\partial T^{q}$ and $\partial U^{q}$ are sufficiently smooth. We define $\Gamma^{q}=$ $X^{q} \backslash\left(\bar{T}^{q} \cup \bar{U}^{q}\right)$ and $\Gamma=\cup_{q \in Q}\{q\} \times \Gamma^{q}$. The initial state (which, in general, is a probability distribution) must lie outside the sets $T$ and $U$. The reset map is defined as a transition measure $R(s, A)$ that defines the probability distribution of the state after the jump and is assumed to be defined so that the system cannot jump directly to $U$ or $T$ [18].

Consider the stopping time $\tau=\inf \{t \geq 0: s(t) \in$ $\partial T \cup \partial U\}$ corresponding to the first hitting time of the boundary of the target or unsafe set. Let $s$ be an initial state in $\Gamma$, then we define the function $V: \bar{\Gamma} \rightarrow \mathbb{R}_{+}$by

$$
V(s)= \begin{cases}E_{s}\left[I_{\left(s\left(\tau^{-}\right) \in \partial T\right)}\right], & s \in \Gamma \\ 1, & s \in \partial T \\ 0, & s \in \partial U\end{cases}
$$

where $E_{s}$ denotes the expectation of functionals given that the initial condition is $s$ and $I$ denotes the indicator function. The function $V(s)$ can be interpreted as the probability that a trajectory starting at $s$ will reach the set $T$ while avoiding the set $U$.

The value function $V$ can be described as the viscosity solution of a system of coupled Hamilton-Jacobi-Bellman (HJB) equations [17], [18]. This function is similar to the value function for the exit problem of a standard stochastic diffusion, but the running and terminal costs depend on the function itself. The coupling between the equations arises because the value function in a particular mode depends on the value function in the adjacent modes and is formally captured by the dependency of the running and terminal costs on the value function $\mathrm{V}$.

Proposition We define a bounded function $c: \bar{S} \rightarrow \mathbb{R}_{+}$ continuous in $x$ such that

$$
c(q, x)= \begin{cases}1, & \text { if } x \in \partial T^{q} \\ 0, & \text { if } x \in \partial U^{q} \cup \partial X^{q}\end{cases}
$$

and denote $\psi^{V}(q, x)=c(q, x)+\int_{\Gamma} V(y) R((q, x), d y)$ and $a(q, x)=\sigma^{\mathrm{T}}(q, x) \sigma(q, x)$. Then, $V$ is the unique viscosity solution of the system of equations

$$
b(q, x) D_{x} V+\frac{1}{2} \operatorname{tr}\left(a(q, x) D_{x}^{2} V\right)=0
$$

in $\Gamma^{q}$ with boundary conditions $V(q, x)=\psi^{V}(q, x)$ on $\partial \Gamma^{q}$.

The proof is a straightforward application of the results presented in [17], [18] to the SHS of the sugar cataract development of the biodiesel production system. 


\section{B. Numerical Methods Based on Dynamic Programming}

One of the advantages of characterizing reachability properties using viscosity solutions is that for computational purposes we can employ numerical algorithms based on discrete approximations. We employ the finite difference method presented in [19] to compute locally consistent Markov chains (MCs). We consider a discretization of the state space denoted by $\bar{S}^{h}=\cup_{q \in Q}\{q\} \times$ $\bar{S}_{q}^{h}$ where $\bar{S}_{q}^{h}$ is a set of discrete points approximating $B^{q}$ and $h>0$ is an approximation parameter characterizing the distance between neighboring points. By the boundness assumption, the approximating MC will have finitely many states which are denoted by $s_{n}^{h}=$ $\left(q_{n}^{h}, \xi_{n}^{h}\right), n=1,2, \ldots, N$. The transition probabilities $p^{h}\left((q, x),\left(q^{\prime}, x^{\prime}\right)\right)$ of the Markov chain are computed to approximate the SHS while preserving local mean and variance [17], [18].

The value function $V$ of the SHS can be approximated by

$$
V^{h}(s)=E_{s}\left[\sum_{n=0}^{\nu_{h}} c\left(q_{n}^{h}, \xi_{n}^{h}\right) I_{\left(n=n_{i}\right)}\right] .
$$

where $\nu_{h}$ is the time the state will enter the target set $T$ or the unsafe set $U$ and $n_{i}$ are the times of the discrete jumps. The function $V^{h}$ can be computed using a value iteration algorithm. The results in [17], [18] show that the algorithm converges for appropriate initial conditions, and further, the solution based on the discrete approximations converges to the one for the original stochastic hybrid system as the discretization becomes finer $(h \rightarrow 0)$. Regarding the efficiency of the computational methods, the iterative algorithm is polynomial in the number of states of the discrete approximation process. Although scalability is a limiting factor, using parallel methods the approach is feasible for realistic systems as biodiesel production system, a seven-dimensional biochemical system for which the approximating process has approximately 500 million states.

\section{EXPERIMENTAL RESULTS}

In this section we present the results of the reachability probability for the SHS biodiesel production model presented in Section 3. The chemical concentration ranges and resolutions used are presented in Table II. We chose the resolution parameters to be similar to the resolution that measurement equipment can achieve in actual experiments.

The resolution parameters for the biodiesel production system result in an MDP with approximately 500 million states. Storing the values at each state alone requires several gigabytes of memory, so we developed a parallel value iteration implementation to improve the performance of the algorithm. The value iteration algorithm is still guaranteed to converge in a parallel implementation as long as updated values are used periodically [4]. Parallel dynamic programming algorithms are well-defined and easy to implement [4]. Our MDP has a regular structure which improves the efficiency of the value iteration algo- rithm and allows us to implement a fairly straitforward partitioning technique for the parallel implementation.

To partition the problem for multiple processors we select five of the seven dimensions of the MDP to divide in half. Each processor only analyzes half of the total range for each of five divided ranges and the entire range for the other two dimensions. The two range divisions in five dimensions create $2^{5}$ or 32 range combinations that must be considered. The processors are each specifically assigned a combination of the ranges to ensure that the entire range for each dimension is computed, and all range values are arranged to minimize communication. Processors with neighboring range values regularly update their neighbors to ensure the value iteration converges.

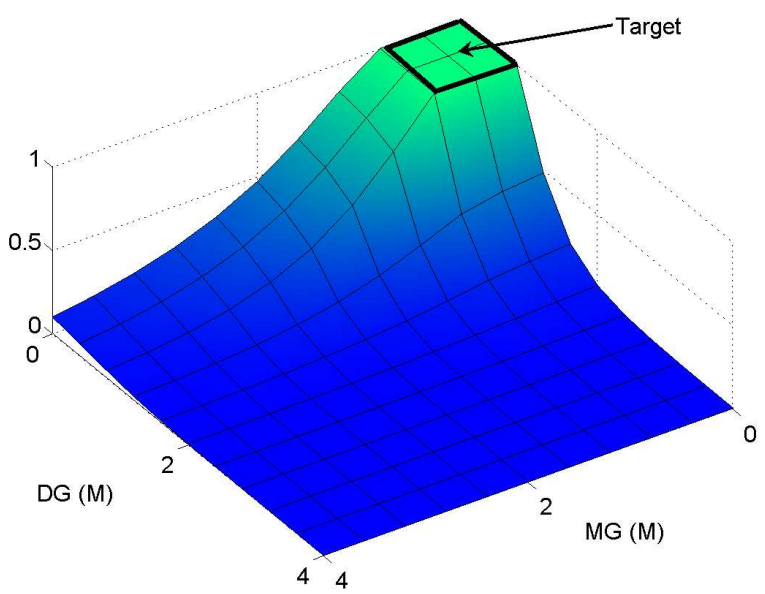

Fig. 2. Projection of the value function (MG,DG) for the temperaturecontrolled reachability results

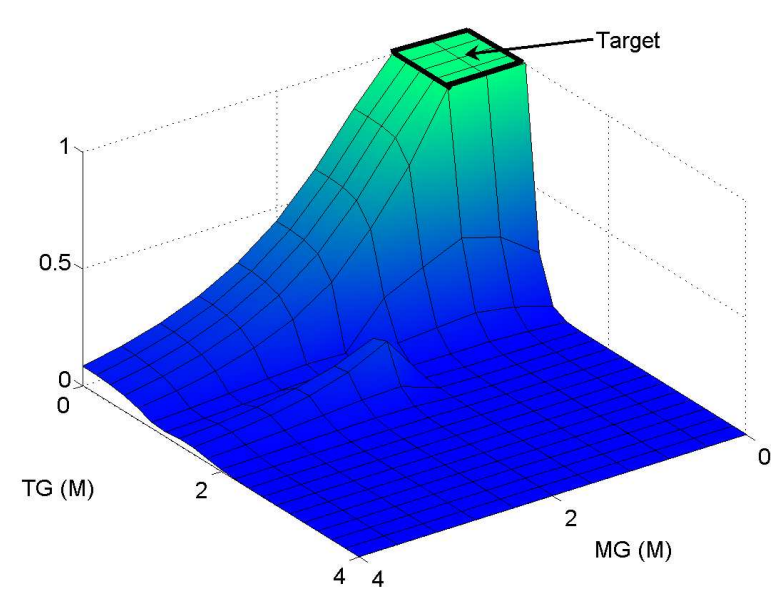

Fig. 3. Projection of the value function (MG,TG) for the temperaturecontrolled reachability results

To visualize our results we plot projections of the data for different concentrations of the chemicals involved. The projection in Figure 2 shows the reachability probability for selected ranges of diglycerides (DG) and monoglycerides $(\mathrm{MG})\left(x_{2}, x_{3}\right)$ where $x_{1}=0.00001, x_{4}=0.00001$, $x_{5}=1.00001, x_{6}=0.5$, and $x_{7}=50.0$. The projection in Figure 3 shows the reachability probability for selected ranges of triglycerides (TG) and monoglycerides (MG) $\left(x_{1}, x_{3}\right)$ where $x_{2}=0.00001, x_{4}=0.00001$, 
$x_{5}=1.00001, x_{6}=1.0$, and $x_{7}=50.0$. Lighter colors represent states which have a higher probability of transitioning to the target set without reaching the unsafe states which are located around the boundary of the state space.

These results indicate that the modeled temperature controller will probably not work effectively for this system because the probability of success for many of the states is fairly low. Further experiments can be performed to determine the ideal temperature to use the heater to maximize efficiency and minimize the use of the heater.

The Advanced Computing Center for Research and Education (ACCRE) at Vanderbilt University provides the parallel computing resources for our experiments (www.accre.vanderbilt.edu). The computers form a cluster of 348 JS20 IBM PowerPC nodes running at $2.2 \mathrm{GHz}$ with 1.4 Gigabytes of RAM per machine. We use $\mathrm{C}++$ as the implementation language because ACCRE supports Message Passing Interface (MPI) compilers for $\mathrm{C}++$. We use the MPI standard for communication between processors because it provides an efficient protocol for message passing middleware for distributed memory parallel computers. The sugar cataract experiment took approximately 2 hours on the 32 processors. Currently, the bottlenecks of this approach are the memory size and speed.

\section{CONCLUSIONS}

Biochemical system modeling and analysis are important but challenging tasks which hold promise to unlock secrets of complicated biochemical systems. SHS are an ideal modeling paradigm for biochemical systems because they incorporate probabilistic dynamics into hybrid systems to capture the inherent stochastic nature of the biochemical systems. The biodiesel production system is excellent example of a system of chemical equations that can be modeled effectively using the presented modeling methods. Our dynamic programming analysis technique provides verification results for realistic systems using parallel computing techniques to lessen the effect of the curse of dimensionality. This technique can be used to design and test controllers of complicated, real-world systems in order to help optimize designs using the proposed verification technique.

Acknowledgements Research is partially supported by the National Science Foundation CAREER grant CNS0347440 .

\section{REFERENCES}

[1] S. Al-Zuhair, The effect of substrate concentrations on the production of biodiesel by lipase-catalysed transesterification of vegetable oils, J. Chem. Tech. and Biotech., vol 81, pp. 299-305, 2006.

[2] R. Alur, C. Belta, F. Ivanicic, V. Kumar, M. Mintz, G. Pappas, H. Rubin, J. Schug, Hybrid Modeling and Simulation of Biomolecular Networks, Hybrid Systems: Computation and Control, LNCS 2034, pp. 19-33, 2001.

[3] R. Barbuti, S. Cataudella, A. Maggiolo-Schettini, P. Milazzo, A. Triona, A Probabilistic Model For Molecular Systems, Fundamenta Informaticae XX, IOS Press, 1-15, 2005.

[4] D. Bertsekas, J. Tsitsiklis, Parallel and Distributed Computation: Numerical Methods, Prentice-Hall, 1989.

[5] H. Blom, J. Lygeros, M. Everdij, S. Loizou, K. Kyriakopoulos, Stochastic Hybrid Systems: Theory and Safety Critical Applications, Lecture Notes in Control and Informations Sciences, Springer, vol.337, 2006.
[6] M. Bujorianu, J. Lygeros, Theoretical Foundations of General Stochastic Hybrid Systems: Modeling and Optimal Control, In Proc. of 43rd IEEE Conf. on Decision and Control, 2004.

[7] Y. Cao, H. Li, L. Petzold, Efficient formulation of the stochastic simulation algorithm for chemically reacting systems, J. Chem. Phys., 121, 4059, 2004.

[8] D. Darnoko, M. Cheryan, Kinetics of Palm Oil Transesterification in a Batch Reactor, JAOCS, Vol 77, no. 12, pp. 1263-1267,2000.

[9] M. Elowitz, A. Levine, E Siggia, P. Swain, Stochastic Gene Expression in a Single Cell, Science 297, 1183, 2002.

[10] R. Ghosh, C. Tomlin, Symbolic Reachable Set Computation of Piecewise Affine Hybrid Automata and its Application to Biological Modeling: Delta-Notch Protein Signalling, Systems Biology, 1:170-183, 2004.

[11] D. Gillespie, A General Method for Numerically Simulating the Stochastic Time Evolution of Coupled Chemical Reactions. J Comp. Phys., 22:403-434, 1976.

[12] E. Haseltine, J. Rawlings, Approximate simulation of coupled fast and slow reactions for stochastic chemical kinetics, J Chem. Phys. 117, 6959, 2002.

[13] J. Hespanha, A. Singh. Stochastic Models for Chemically Reacting Systems Using Polynomial Stochastic Hybrid Systems, Int. J. on Robust Control, Special Issue on Control at Small Scales, 15:669689, 2005.

[14] J. Hu, M. Prandini, and S. Sastry, Probabilistic Safety Analysis in Three Dimensional Aircraft Flight, Proc. of 42nd IEEE Conf. on Decision and Control, pp. 5335-5340, 2003.

[15] J. Hu, W. Wu, and S. Sastry, Modeling Subtilin Production in Bacillus subtilis Using Stochastic Hybrid Systems, Hybrid Systems: Computation and Control 2004, LNCS, vol. 2993, pp. $417-$ 431, 2004.

[16] X. Koutsoukos, Optimal Control of Stochastic Hybrid Systems Based on Locally Consistent Markov Decision Processes, Int. J. of Hybrid Systems, 4, 301-318, 2004.

[17] X. Koutsoukos and D. Riley, Computational Methods for Reachability Analysis of Stochastic Hybrid Systems, Hybrid Systems: Computation and Control 2006 LNCS 3927, pp. 377-391, 2006.

[18] X. Koutsoukos and D. Riley, Computational Methods for Verification of Stochastic Hybrid Systems, IEEE Transactions on Systems, Man, and Cybernetics - Part A. To appear.

[19] H. Kushner, P. Dupuis, Numerical Methods for Stochastic Control Problems in Continuous Time, Springer, 2001.

[20] H. Noureddini, D. Zhu, Kinetics of Transesterification of Soybean Oil, JAOCS, vol 74, no. 11, pp. 1457-1463, 1997.

[21] M. Puterman, Markov Decision Processes-Discrete Stochastic Dynamic Programming, Wiley: Hoboken, New Jersey, 2005.

[22] H. Salis and Y. Kaznessis, Accurate hybrid stochastic simulation of a system of coupled chemical or biochemical reactions, J. Chem. Phys., v122, pp. 54-103, 2005.

[23] R. Sridharan, I. Mathai, Transesterification Reactions, J. Sri. Ind. Res. Vol. 33, pp 178-187, 1974.

[24] Y. Zhang, M. Dube, D. McLean, M. Kates, Biodiesel Production from Waste Cooking Oil: 1 Process Design and Technological Assessment, Bioresource Technology, vol. 89, pp. 1-16, 2003. 